\title{
Correlation of Gut Firmicutes/Bacteroidetes Ratio with Fibrosis and Steatosis in Patients with Non- alcoholic Fatty Liver Disease
}

Chyntia Olivia Maurine Jasirwan ( $\sim$ chynmadu@gmail.com )

Hepatobilary Division https://orcid.org/0000-0001-5950-8245

Akhmadu Muradi

Rumah Sakit Dr Cipto Mangunkusumo

Irsan Hasan

Rumah Sakit Dr Cipto Mangunkusumo

Marcellus Simadibrata

Rumah Sakit Dr Cipto Mangunkusumo

Ikhwan Rinaldi

Rumah Sakit Dr Cipto Mangunkusumo

\section{Cosmas Rinaldi A Lesmana}

Rumah Sakit Dr Cipto Mangunkusumo

Andri Sanityoso Sulaiman

Rumah Sakit Dr Cipto Mangunkusumo

Juferdy Kurniawan

Rumah Sakit Dr Cipto Mangunkusumo

Kemal Fariz Kalista

Rumah Sakit Dr Cipto Mangunkusumo

Saut Horas Nababan

Rumah Sakit Dr Cipto Mangunkusumo

Rino Alvani Gani

Rumah Sakit Dr Cipto Mangunkusumo

Research article

Keywords: NAFLD, Ratio of Firmicutes/Bacteroidetes, Dysbiosis of gut microbiota

Posted Date: April 22nd, 2020

DOI: https://doi.org/10.21203/rs.3.rs-23643/v1 
License: (c) (i) This work is licensed under a Creative Commons Attribution 4.0 International License. Read Full License 


\section{Abstract}

Background: We investigated the gut microbiota in patients with non-alcoholic fatty liver disease (NAFLD) and its correlation with fibrosis and steatosis as reflected in the controlled attenuation parameter and transient elastography values

Methods : A cross-sectional study was performed on 37 patients with NAFLD at Cipto Mangunkusumo National General Hospital from December 2018 to March 2019. The gut microbiota was investigated in fecal samples with 16S RNA sequencing using the next-generation sequencing platform MiSeq (Illumina).

Results : NAFLD was more common in patients with metabolic syndrome. Firmicutes, Bacteroidetes, and Proteobacteria were the predominant phyla. Bacteroides was more dominant than Prevotella, contrary to the results in previous studies on normal populations in Indonesia. Microbiota dysbiosis was observed in most samples. The gastrointestinal microbiota diversity was significantly decreased in patients with NAFLD with high triglyceride levels and central obesity. The Firmicutes/Bacteroidetes ratio correlated with steatosis and obesity, whereas some other species in the lower taxonomy were mostly correlated with steatosis and obesity without fibrosis. Proteobacteria is the only phylum strongly correlated with fibrosis in patients with normal body mass index.

Conclusions : The gut microbiota diversity was decreased in patients with NAFLD with high triglyceride levels and central obesity, and certain gut microbes were correlated with fibrosis and steatosis.

\section{Background}

Non-alcoholic fatty liver disease (NAFLD) is a fatty liver condition that can lead to hepatocellular carcinoma (HCC) and has globally become a major health problem with a prevalence of $25.24 \%$ ( $95 \% \mathrm{Cl}$ : 22.10-28.65) [1]. However, Hasan et al. (2002) first reported NAFLD prevalence at 30.6\% from 965 subjects in West Java, Indonesia [2]. Diet, insulin resistance, and metabolic syndrome have key roles in NAFLD pathogenesis, resulting in complex multifactorial disease $[3,4]$. Gut microbiota were also found to differ in the NAFLD population, yet the mechanism for this is unclear. The change in gut microbiota increases fatty acid absorption and the number of inflammasomes and pro-inflammatory cytokines in the blood $[5,6]$.

There are only four major phyla in the human intestinal microbiome: Firmicutes, Bacteroidetes, Actinobacteria, and Proteobacteria, $90 \%$ of which are dominated by gram-positive Firmicutes and gramnegative Bacteroidetes [7]. Some studies have used the ratio of the two dominant phyla (Firmicutes and Bacteroidetes) as a marker for microbial dysbiosis [8-10]. Change in this ratio have also been found in several metabolic disorders [9].

A study conducted by Zhu et al. (2013) reported that the gut microbiota composition in patients with nonalcoholic steatohepatitis (NASH) and obesity when compared with that of the normal control subjects showed a certain pattern related to disease progression. They observed increased abundance of alcohol- 
producing bacteria in the microbiomes of patients with $\mathrm{NASH}$, which suggested the relationship between gut microbiota composition and the severity of NAFLD [11]. Lelouvier et al. (2016) found specific differences in the proportion of several bacterial taxa in both blood and feces that correlate with the presence of liver fibrosis, thus defining a specific signature of the liver disease, but this study did not show any correlation with liver steatosis [12].

The microbiota in patients with NAFLD experience changes in composition associated with dysbiosis, loss of gut barrier integrity, and an increase in pro-inflammatory immune responses, which are responsible for NAFLD pathogenesis and progression [6]. Some studies on the microbiota profile of patients with NAFLD and healthy controls identified microbiota at different taxonomy levels. However, the current studies are inconsistent in determining the association between microbiota profile and NAFLD, although it is thought that there is a relationship between microbiome composition and host health $[13,14]$. The inconsistent results were probably due to variable gut microbiota composition across geographically distinct populations.

The relationship between the Firmicutes/Bacteroidetes ratio (as dysbiosis marker) and the severity of NAFLD, especially in Indonesia, is still unknown. The microbiota composition can be a roadblock in NAFLD management, especially intervention at the gut microbiota level. The goal of this study was to investigate the configuration of gut microbiota in patients with NAFLD and its correlation with fibrosis and steatosis condition as reflected in the controlled attenuation parameter (CAP) and transient elastography (TE) values. TE (Fibroscan ${ }^{\circledR}$ Echosens) measures the velocity of the sound wave passing through the liver and then converts that measurement into a liver stiffness measurement; the entire process is often referred to as liver ultrasonographic (USG) elastography. The CAP specifically targets liver steatosis using a process based on TE. It measures the degree of ultrasound attenuation by hepatic fat at the central frequency of the Fibroscan $\circledast \mathrm{M}$ probe simultaneously with liver stiffness measurement.

\section{Methods}

This study used correlation tests to evaluate the relationship between two numerical variables. First, the gut microbiota were profiled in a cohort of patients with NAFLD. The correlation between the Firmicutes/Bacteroidetes ratio and CAP and TE values in patients with NAFLD were analyzed. Afterwards, the correlation of each microorganism with CAP and TE values were also analyzed to determine if certain microbes were correlated with the degree of fibrosis and steatosis in NAFLD. All the experiments were performed in accordance with relevant guidelines and regulations.

\section{Subjects}

The study samples were taken from patients at the Hepatobiliary Division Cipto Mangunkusumo Hospital Jakarta from December 2018 to March 2019. The sampling method used simple random sampling with a numeric correlation test formula, which determined that the number of samples needed was 37 subjects, including drop out possibilities. Patients with NAFLD between 18 and 60 years old, who were willing to 
participate in the study, were included, and informed consent from the patients was obtained. The exclusion criteria were pregnant or lactating patients, patients with other chronic liver diseases [hepatitis $B$, hepatitis $C$, autoimmune hepatitis, or with history of alcohol consumption ( $>40 \mathrm{~g} /$ day)], patients with a history of intestinal resection surgery, patients with chronic intestinal inflammation [inflammatory bowel disease (IBD)], patients with liver cell carcinoma, patients with a history of antibiotics and probiotics in the past month, or patients with a special diet in the past month as evidenced by a food recall questionnaire. The detailed flow chart of subject recruitment are shown in Figure 1.

\section{Measurements}

Patients filled out the informed consent form prior to examinations and underwent CAP and TE examinations using Fibroscan ${ }^{\circledR}$ Echosens. The ultrasonographic probe was used to measure the level of fibrosis (in $\mathrm{kPa}$ ) and steatosis (in $\mathrm{dB} / \mathrm{m}$ ). Patients were also told to collect stool into the provided sterile tubes. The stool samples were initially stored at $2^{\circ} \mathrm{C}-8^{\circ} \mathrm{C}$ and then immediately taken to be stored at $-80^{\circ} \mathrm{C}$ within 4 hours after sample collection. Afterwards, the bacterial genome was extracted at the laboratory of Child Health Department and sequenced by Biosains Medika (BioSM) Indonesia using the stool DNA kit Herculase II Fusion and the DNA Polymerase Nextera XT Index Kit V2 with 16S Metagenomic Sequencing Library Preparation Part \#15044223 Rev. B protocols. The 16S rRNA sequencing used NGS platform MiSeq (Illumina) with four steps: sample preparation, library construction, sequencing and raw data collection.

\section{Statistical Analysis}

The data were analyzed using IBM SPSS Indonesia statistical program version 23. Numerical data normality was examined using a Shapiro-Wilk test. Data were not normally distributed if the $p$ value < 0.05. The Spearman correlation test was used to evaluate the correlation between the Firmicutes/Bacteroidetes ratio and CAP and TE values. The correlation coefficient was interpreted with an $r$ value and considered significant if $p<0.05$ and with the following interpretation: $0-0.19=$ very weak, $0.2-0.39=$ weak, $0.4-0.59=$ medium, $0.6-0.79=$ strong and $0.8-1=$ very strong correlation .

Upon correlation between variables, we continued to search for the intersecting value by creating a receiver operating curve. Results obtained as the area under the curve values can be interpreted as follows: $0.5-0.6=$ very low accuracy, $0.6-0.7=$ low accuracy, $0.7-0.8=$ medium accuracy, $0.8-0.9=$ high accuracy and 0.9-1.0 = very high accuracy. The microbiota diversity test between the group with significant fibrosis compared with non-significant fibrosis and significant steatosis compared with nonsignificant steatosis used the Mann-Whitney test. Dysbiosis was determined by an intersecting value between the quartile of the Firmicutes/Bacteroidetes ratio to fibrosis and steatosis and/or based on the intersecting value of the diversity value of fibrosis or steatosis group.

\section{Results}


The characteristics of the 37 subjects are presented in Table 1. Women dominated the group as 23 of them were female while only 14 were male with an average age of $50 \pm 7.93$ years old. The body mass index (BMI) assessment showed that 25 subjects were obese, seven were overweight, and five had normal BMI. The average waist circumference was $96.65 \mathrm{~cm}$, with 24 subjects experiencing dyslipidemia, and 30 subjects had type 2 diabetes mellitus. However, based on the HbA1c median value of 6.6 (4.814 ), we can see that the blood glucose was under control as also shown by the moderate value of fasting blood glucose and 2-hour postprandial blood glucose at 108 and $149 \mathrm{mg} / \mathrm{dL}$, respectively. The lipid profile, including triglyceride, low-density lipoprotein (LDL), and high-density lipoprotein (HDL) levels also showed moderate values, while the liver function test (aspartate aminotransferase, alanine aminotransferase, and serum albumin) also appeared normal.

We divided the subjects into three groups based on the BMI: normal (5 subjects), pre-obesity (7 subjects), and obesity (25 subjects). The characteristics of each group are presented in Table 2 . In all three groups, most patients had central obesity (normal $4 / 5$, pre-obesity $5 / 7$ and obesity $25 / 25$ ). Dyslipidemia was dominant in the pre-obesity and obesity groups ( $5 / 7$ and $18 / 25$, respectively), while $4 / 5$ in the normal BMI group did not have dyslipidemia. Diabetes mellitus was dominant in all groups, even in the normal BMI group. The prevalence of diabetes mellitus in the pre-obesity and obesity group was $71.4 \%$ and $80 \%$, respectively.

\section{Intestinal Microbiota}

\section{Characteristics of Intestinal Microbiota in Bacterial Mean Relative Abundance Based on Taxonomy}

Intestinal microbiota were dominated by three major phyla: Firmicutes $55.35 \%$; Bacteroidetes $29.94 \%$ and Proteobacteria $11.83 \%$ (Figures 2 and 3 ). The majority of subjects (20 of 37 subjects) had been dominated by Firmicutes, while 17 others were dominated by Bacteroidetes.

\section{Microbiota Diversity Index}

Table 3 shows the Mann-Whitney test between alpha microbiota diversity index with other parameters, including fibrosis, steatosis, BMI, central obesity, triglyceride, HDL, LDL, and diabetes mellitus. The analysis presents the quantity of bacteria for each taxonomy level in its community. The diversity was determined by richness or amount (how numerous is one component was found in one population) and evenness or distribution (comparison of the amount of component toward the number of other components in one population). The alpha diversity used the Shannon-Weaver (H) Index or Simpson (D) Diversity Index. Both indices measure the richness and evenness of microbiota, but the Shannon index focuses on richness while the Simpson index focuses on evenness. Both used phylotype data or operational taxonomic units (OTU), which are clusters of similar DNA sequences to be later identified with the database. The alpha diversity was significantly different in the central obesity group. All three diversity tests (OTU, Shannon index, and inverse Simpson index) were significantly different between the increased and non-increased triglyceride group ( $p=0.006$ vs. 0.008 vs. 0.0037 ). 


\section{Correlation Between Intestinal Microbiota with Fibrosis and Steatosis in NAFLD Patients based on Body Mass Index}

Table 4 shows the Spearman correlation between the Firmicutes/Bacteroidetes ratio with each microbiota based on BMI. If divided into groups based on $\mathrm{BMI}$, the medium positive correlation was between the Firmicutes/Bacteroidetes ratio with steatosis $(r=0.435 ; p=0.030)$ and in the obesity group only. At the phylum level, the medium positive correlation $(r=0.528 ; p=0.007)$ was also found between Firmicutes and steatosis. The only phylum strongly correlated with fibrosis in the normal BMI group was Proteobacteria $(r=0.921 ; p=0.026)$. In the pre-obesity $B M I$ group, there is a very strong positive correlation between steatosis with Lachnospiraceae $(r=0.883 ; p=0.008)$ and Intestinimonas butyriciproducens $(r=0.847 ; p=0.016)$.

\section{Discussion}

\section{Study Subject Characteristics}

There were more women than men in this study, similar to a population-based study in Thailand by Summart (2017) [15]. Metabolic syndrome was dominant in the study subjects, which aligned with many other studies stating an apparent relationship among NAFLD, obesity, diabetes mellitus, and metabolic syndrome. In this study, 25 out of 37 subjects were obese, and 30 from 37 subjects had type 2 diabetes mellitus. Such characteristics were similar to other previous studies, which stated that there is a higher prevalence of NAFLD in adults with obesity (65.7\%) and type 2 diabetes mellitus (74\%) $[16,17]$. Individuals with NAFLD have a five times higher risk of developing diabetes [18,19]. The association between NAFLD and type 2 diabetes mellitus can be explained through insulin resistance, dyslipidemia, and accumulation of liver triglyceride in NAFLD and $\beta$-cell defect in type 2 diabetes mellitus [20].

Although there were many previous studies on the microbiota, the results were inconsistent. A study by Raman et al. (2013) reported an increase in Firmicutes in obese patients with NAFLD compared with that of the patients without obesity and NALD [21]. Another study by Jiang et al. (2015) found no significant microbiota differences in NAFLD and normal control group [22]. Our study found that on average, Firmicutes was higher than Bacteroidetes. While at the genus level, Bacteroides (14.43\%) were more numerous than Prevotella (9.14\%), confirming that Bacteroides dominated other genera from the Firmicutes phylum.

Rahayu (2019) studied young Indonesian adult microbiota profile [23] and reported that in numerous orders, the microbiota was Clostridium, Prevotella, Atopobium, Bifidobacterium, and Bacteroides. The results were quite similar to other local studies showing dominant Prevotella but different from our study. This may be due to the different study population as the subjects in this study lived in Jakarta and represented the urban population with high protein and animal fat in their diet [24].

We attempted to identify the prevalence of dysbiosis by looking at the diversity of microbiota and/or an increase in the ratio of Firmicutes/Bacteroidetes. Using dysbiosis criteria of Firmicutes/Bacteroidetes 
ratio, there were 26 out of 37 subjects with dysbiosis. There were 25 subjects fulfilling the criteria if only based on the decrease of microbiota diversity. By combining the two criteria, we found dysbiosis in 18 subjects.

\section{Microbiota Diversity Index in NAFLD}

The microbiota diversity in our study was assessed using the alpha diversity index through OTUs, the Shannon index or the inverse Simpson index. The results showed significant differences in microbial diversity between the central obesity and non-central obesity group and between the high triglyceride and normal triglyceride group. Central obesity and high triglyceride groups showed a reduction in diversity compared with that of the other groups. This is similar with studies by Turnbaugh et al. (2009) [25] and Le Chatelier (2013) [26], which showed a total reduction in bacteria diversity in obesity. This is also in concordance with the dysbiosis theory commonly used in many studies as a marker for dysbiosis condition related to diseases.

\section{Correlation of Firmicutes/Bacteroidetes with Fibrosis and Steatosis Based on Body Mass Index}

We analyzed the correlation between the Firmicutes/Bacteroidetes ratio and microbiota in each taxonomy level with fibrosis and steatosis based on BMI. NAFLD analysis in each BMI group showed that the Firmicutes/Bacteroidetes ratio only had a positive correlation with steatosis in the obesity group. There was no significant correlation with fibrosis and also with steatosis in groups other than the obesity group. We also found that Firmicutes had a strong positive correlation with steatosis in the obesity group. This is similar to many previous studies that highlighted the role of Firmicutes in obesity $[8,21]$.

Further analysis in lower taxonomy level in each microbiota revealed that only the group from phylum Proteobacteria was correlated with fibrosis in the obesity and normal BMI group. This is similar to a study by Loomba et al. (2017) [27]. The higher the fibrosis degree, the more numerous Proteobacteria and Bacteroidetes. Through this correlation study, we can see that Proteobacteria has a role in the process of liver fibrosis, although the exact mechanism is still unknown.

Most microbiotas had a positive correlation with steatosis, especially in obese patients. Some of which were from the order Clostridiales and Selemonodales in the Firmicutes phylum. While those correlated with steatosis in the normal BMI group were from the Actinobacteria phylum, the mechanism underlying steatosis by the microbiota were from several pathways especially related to fat metabolism [25]. Lactobacillus was very consistent in protecting steatosis, while the Enterobacteriaceae family in our study showed a very strong positive correlation with steatosis in the normal BMI group. These findings differed from those of previous studies [11]. However, in a study by Rahayu in healthy Indonesian population showed that the family of Enterobacteriaceae, especially Escherichia coli is part of the normal flora that increases in old age [23].

At present, there are very few studies that can show the direct cause and effect relationship between microbiota and NAFLD pathogenesis. However, some interventional studies in animals showed the 
important role of intestinal microbiota, especially in triggering a metabolic response. The intestinal microbiota from obese subjects can induce liver steatosis through modulation of fat metabolism. This is probably why most intestinal microbiota in our study correlated with steatosis but not with fibrosis. The process of turning steatosis into fibrosis needs more complex pathways and involving more factors aside from intestinal microbiota [28].

We acknowledge that the limitations of this study include the small sample size because of which we could not demonstrate that small variations in the bacterial counts were statistically significant. However, this cross-sectional single-center study was unable to view in detail the change in microbiota in relation to disease progression. We did not use normal healthy control because it was difficult to find a population in urban settings that was absolutely healthy and free of metabolic disorders and was not affected by extreme diet.

In conclusion, we assumed that the bigger the difference between the subgroups studied, the stronger the potential effect of the bacteria on the phenotype. This is the first study in Indonesia to thoroughly profile the microbiota in patients with NAFLD using next-generation sequencing and tried to find the correlation of each microbiota with fibrosis and steatosis. There was a strong positive correlation between the Firmicutes/Bacteroidetes ratio with steatosis in the obesity group. There were positive and negative correlations between some microbiota with fibrosis and steatosis. We suggest that future studies examine microbiota profiles in the general Indonesian population, the microbiota population in patients with NAFLD based on groups with other metabolic syndrome co-morbidities and the relationship between microbiota metabolism products and NAFLD.

\section{Abbreviations}

BMI : Body Mass Index

CAP : Controlled Atenuation Parameter

DNA : Deoxyribonucleic Acid

HDL : High Density Lipoprotein

IBD : Inflammatory Bowel Disease

LDL : Low Density Lipoprotein

NAFLD : Nonalcoholic Fatty Liver Disease

NASH : Nonalcoholic Steatohepatitis

OTU: Operational Taxonomix Units

RNA: Ribonucleic Acid 
TE : Transient Elastography

USG : Ultrasonographic

\section{Declarations}

\section{Ethics approval and consent to participate}

All procedures performed in studies involving human participants were in accordance with the ethical standards of the institutional and/or national research committee and with the 1964 Helsinki declaration and its later amendments or comparable ethical standards.

This study has been approved by IRB/Institutional Review Board (Medical Faculty Universitas Indonesia Ethical Committee) no. 1336/UN2.F1/ETIK/2018.

Written Informed consent was obtained from all the study subjects.

\section{Consent for Publication}

Not applicable

\section{Availability of data and materials}

The data that support the findings of this study are available on request with the corresponding author. The data are not publicly available because they contain information that could compromise research participants' privacy/consent.

\section{Competing interests}

The authors declare that they have no competing interests

\section{Funding}

This study received one third funding from the university (Medical Faculty Universitas Indonesia) and the rest from the private funding.

\section{Author Contributions}

COMJ proposed and conducted the study. AM, IH, MS, and IR performed the research and supervised the study. CRAL provided the idea for the first draft of the manuscript. ASS, JK, KFK, SHN, and RAG collected and analyzed the data. All authors contributed to the design of the study, interpretation of the results, and in writing the final manuscript. Chyntia Olivia Maurine Jasirwan is the guarantor of this study.

\section{Acknowledgements}


We thank to Ms. Anugrah Dwi Handayu for helping of stool collection and PCR analyses, and Ms. Gita who helped in datasets and analyses.

\section{References}

1. Younossi ZM, Koenig AB, Abdelatif $D$, et al. Global epidemiology of nonalcoholic fatty liver diseaseMeta-analytic assessment of prevalence, incidence, and outcomes. Hepatology. 2016;64:73-84.

2. Hasan IGR, Machmud R. Prevalence and risk factors for nonalcoholic fatty liver in Indonesia. J Gastroenterol Hepatol. 2002;17:A30.

3. Shulman GI. Cellular mechanisms of insulin resistance. J Clin Invest. 2000;106:171-6.

4. Tarantino G, Finelli C. What about nonalcoholic fatty liver disease as a new criterion to define metabolic syndrome? World J Gastroenterol. 2013;19:3375-84.

5. Bugianesi E, Moscatiello S, Ciaravella MF, et al. Insulin resistance in nonalcoholic fatty liver disease. Curr Pharm Des. 2010;16:1941-51.

6. Kirpich IA, Marsano LS, McClain CJ. Gut-liver axis, nutrition, and non-alcoholic fatty liver disease. Clin Biochem. 2015;48:923-30.

7. Mokhtari Z, Gibson DL, Hekmatdoost A. Nonalcoholic fatty liver disease, The gut microbiome, and diet. Adv Nutr. 2017;8:240-52.

8. Ley RE, Turnbaugh PJ, Klein S, et al. Microbial ecology: human gut microbes associated with obesity. Nature 2006;444:1022-3.

9. Mariat D, Firmesse O, Levenez F, et al. The Firmicutes/Bacteroidetes ratio of the human microbiota changes with age. BMC Microbiol. 2009;9:123.

10. Yang T, Santisteban MM, Rodriguez V, et al. Gut dysbiosis is linked to hypertension. Hypertension 2015;65:1331-40.

11. Zhu L, Baker SS, Gill C, et al. Characterization of gut microbiomes in nonalcoholic steatohepatitis (NASH) patients: a connection between endogenous alcohol and NASH. Hepatology 2013;57:601-9.

12. Lelouvier B, Servant F, Païssé $S$, et al. Changes in blood microbiota profiles associated with liver fibrosis in obese patients: a pilot analysis. Hepatology 2016;64:2015-27.

13. Lau E, Carvalho D, Freitas P. Gut microbiota: association with NAFLD and metabolic disturbances. BioMed Res Int. 2015; 2015:979515.

14. Mouzaki M, Comelli EM, Arendt BM, et al. Intestinal microbiota in patients with nonalcoholic fatty liver disease. Hepatology. 2013;58:120-7.

15. Summart U, Thinkhamrop B, Chamadol N, et al. Gender differences in the prevalence of nonalcoholic fatty liver disease in the Northeast of Thailand: a population-based cross-sectional study. F1000Res. 2017;6:1630.

16. Williams CD, Stengel J, Asike MI, et al. Prevalence of nonalcoholic fatty liver disease and nonalcoholic steatohepatitis among a largely middle-aged population utilizing ultrasound and liver biopsy: a prospective study. Gastroenterology 2011;140:124-31. 
17. Paquissi FC. Immune imbalances in non-alcoholic fatty liver disease: from general biomarkers and neutrophils to interleukin-17 axis activation and new therapeutic targets. Front Immunol. 2016;7:490.

18. Jäger $S$, Jacobs $S$, Kröger $J$, et al. Association between the fatty liver index and risk of type 2 diabetes in the EPIC-Potsdam Study. PLOS ONE 2015;10:e0124749.

19. Hazlehurst JM, Woods C, Marjot T, et al. Non-alcoholic fatty liver disease and diabetes. Metabolism. 2016;65:1096-108.

20. Forlani G, Giorda C, Manti R, et al. The burden of NAFLD and its characteristics in a nationwide population with type 2 diabetes. J Diabetes Res. 2016;2931985.

21. Sohail MU, Althani A, Anwar $\mathrm{H}$, et al. Role of the gastrointestinal tract microbiome in the pathophysiology of diabetes mellitus. J Diabetes Res. 2017;9631435.

22. Jiang $W$, Wu N, Wang $X$, et al. Dysbiosis gut microbiota associated with inflammation and impaired mucosal immune function in intestine of humans with non-alcoholic fatty liver disease. Sci Rep. 2015;5:8096.

23. Rahayu ES, Utami T, Mariyatun M, et al. Gut microbiota profile in healthy Indonesians. World J Gastroenterol. 2019;25:1478-91.

24. Daya M, Pujianto DA, Witjaksono F, et al. Obesity risk and preference for high dietary fat intake are determined by FTO rs9939609 gene polymorphism in selected Indonesian adults. Asia Pac J Clin Nutr. 2019;28:183-91.

25. Turnbaugh PJ, Bäckhed F, Fulton L, et al. Diet-induced obesity is linked to marked but reversible alterations in the mouse distal gut microbiome. Cell Host Microbe 2008;3:213-23.

26. Le Chatelier E, Nielsen T, Qin J, et al. Richness of human gut microbiome correlates with metabolic markers. Nature 2013;500:541-6.

27. Loomba R, Seguritan V, Li W, et al. Gut microbiome-based metagenomic signature for non-invasive detection of advanced fibrosis in human nonalcoholic fatty liver disease. Cell Metab. 2017;25: 105462.

28. Wieland A, Frank DN, Harnke B, et al. Systematic review: microbial dysbiosis and nonalcoholic fatty liver disease. Aliment Pharmacol Ther. 2015;42:1051-63.

\section{Tables}

Table 1. Characteristics of research subjects 


$\begin{array}{lr}\text { Gender, } \mathrm{n} & 23 / 37 \\ \text { Female } & 14 / 37 \\ \text { Male } & 50 \pm 7.93 \\ \text { Age (years), mean } \pm \text { SD }\end{array}$

BMI $\left(\mathrm{kg} / \mathrm{m}^{2}\right)$

Normal (18.5-24.9)

Pre-obesity (25.0-29.9)

$7 / 37$

Obesity ( $\geq 25$ )

$25 / 37$

Waist circumference $(\mathrm{cm})$, mean \pm SD

$96.65 \pm 10.02$

Fasting blood glucose (mg/dL), median (range)

$108(51-291)$

2 hour postprandial blood glucose (mg/dL), median (range)

149 (76-473)

HbA1c, median (range)

$6.6(4.8-14)$

Triglyceride, median (range)

$124(73-282)$

HDL (mmol/L), median (range)

$45(29-111)$

LDL (mmol/L), median (range)

$129.43 \pm 39.09$

Dyslipidemia, n (\%)

No

$13 / 37$

Yes

$24 / 37$

Type 2 diabetes mellitus, $\mathrm{n}(\%)$

No $7 / 37$

Yes

$30 / 37$

AST (U/L), median (range)

$20(12-78)$

ALT (U/L), median (range)

$19(10-61)$ 
SD: standard deviation; BMI: body mass index; HDL, high-density lipoprotein cholesterol; LDL, low-density lipoprotein cholesterol; AST, aspartate aminotransferase; ALT, alanine aminotransferase; HbA1c, Hemoglobin A1c 
Table 2. Demographic Data and Clinical Characteristics of Study Subjects Based in Body Mass Index

\begin{tabular}{|c|c|c|c|}
\hline \multirow[t]{2}{*}{ Variable } & \multicolumn{3}{|c|}{ BMI } \\
\hline & Normal $(n=5)$ & Pre-obesity $(n=7)$ & Obesity $(n=25)$ \\
\hline \multicolumn{4}{|l|}{ Gender } \\
\hline Female & $3 / 5$ & $3 / 7$ & $17 / 25$ \\
\hline Male & $2 / 5$ & $4 / 7$ & $8 / 25$ \\
\hline \multicolumn{4}{|c|}{ Central Obesity } \\
\hline No & $1 / 5$ & $2 / 7$ & $0 / 25$ \\
\hline Yes & $4 / 5$ & $5 / 7$ & $25 / 25$ \\
\hline \multicolumn{4}{|l|}{ Dyslipidemia } \\
\hline No & $4 / 5$ & $2 / 7$ & $7 / 25$ \\
\hline Yes & $1 / 5$ & $5 / 7$ & $18 / 25$ \\
\hline \multicolumn{4}{|l|}{ Type 2 DM } \\
\hline No & $0 / 5$ & $2 / 7$ & $5 / 25$ \\
\hline Yes & $5 / 5$ & $5 / 7$ & $20 / 25$ \\
\hline \multicolumn{4}{|l|}{ Triglyceride } \\
\hline Normal & $1 / 5$ & $2 / 7$ & $8 / 25$ \\
\hline Increased & $4 / 5$ & $5 / 7$ & $17 / 25$ \\
\hline \multicolumn{4}{|l|}{ LDL } \\
\hline Normal & $2 / 5$ & $6 / 7$ & $20 / 25$ \\
\hline Increased & $3 / 5$ & $1 / 7$ & $5 / 25$ \\
\hline \multicolumn{4}{|l|}{ HDL } \\
\hline Normal & $3 / 5$ & $3 / 7$ & $13 / 25$ \\
\hline Low & $2 / 5$ & $4 / 7$ & $12 / 25$ \\
\hline
\end{tabular}

BMI: body mass index; Type 2 DM: type 2 diabetes mellitus; HDL, high-density lipoprotein cholesterol; LDL, low-density lipoprotein cholesterol

Table 3. Correlation of intestinal microbiota with fibrosis and steatosis according to the BMI of patients with NAFLD 


\begin{tabular}{|c|c|c|c|c|c|c|c|c|c|c|c|}
\hline \multirow[t]{4}{*}{ Microbiota } & \multicolumn{3}{|c|}{$\begin{array}{l}\text { Normal } \\
(n=5)\end{array}$} & \multicolumn{4}{|c|}{$\begin{array}{l}\text { Pre-obese } \\
\qquad(n=7)\end{array}$} & \multicolumn{4}{|c|}{$\begin{array}{l}\text { Obese } \\
(n=25)\end{array}$} \\
\hline & Fibrosis & \multicolumn{2}{|c|}{ Steatosis } & \multicolumn{2}{|c|}{ Fibrosis } & \multicolumn{2}{|c|}{ Steatosis } & \multicolumn{2}{|c|}{ Fibrosis } & \multicolumn{2}{|c|}{ Steatosis } \\
\hline & $\mathbf{P}$ & $\mathbf{r}$ & $\mathrm{P}$ & $\mathbf{r}$ & $P$ & $\mathbf{r}$ & $\mathbf{P}$ & $\mathbf{r}$ & $\mathbf{P}$ & $\mathbf{r}$ & $P$ \\
\hline & value & & value & & value & & value & & value & & value \\
\hline Firmicutes & -0.6670 .219 & 0.700 & 0.188 & -0.35 & 70.432 & -0.072 & 20.878 & 0.235 & 0.257 & 0.528 & 0.007* \\
\hline Bacteroidetes & 0.4100 .493 & 0.000 & 1.000 & -0.07 & 10.879 & 0.234 & 0.613 & -0.106 & 60.613 & $-0.22 \varepsilon$ & 80.272 \\
\hline Proteobacteria & $0.9210 .026 *$ & -0.872 & 0.054 & 0.222 & 0.632 & 0.505 & 0.248 & -0.104 & 40.621 & -0.308 & 80.134 \\
\hline Actinobacteria & -0.1030 .870 & -0.100 & 0.873 & 0.00 & 1.000 & -0.505 & 50.248 & 0.360 & 0.077 & 0.275 & 0.184 \\
\hline Firmicutes/Bacteroidetes Ratio & -0.6670 .219 & 0.400 & 0.505 & -0.17 & $90.702-$ & -0.523 & 30.229 & 0.250 & 0.228 & 0.435 & $0.030 *$ \\
\hline
\end{tabular}

Firmicutes/Negativicutes/Selemonadales

Selemonadaceae/Megamonas

Acidaminococcaceae/Phascolarcto

Veillonellaceae/Dialister/D. succinatiphilus

Veillonellaceae/Megashphaera/M. elsdenii

Selenomonadaceae/Megamonas/M. funiformis

Firmicutes/-/Erysipelotrichales

Erysipelotrichaceae/Holdemania/H. massiliensis

Firmicutes/Bacilli/Lactobacillales

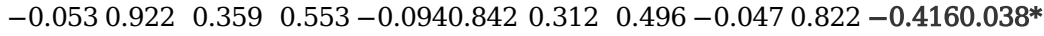

$0.205 \quad 0.741-0.1000 .8730 .5590 .192-0.2000 .667 \quad 0.091 \quad 0.664-0.4690 .018^{*}$

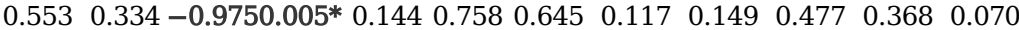

$\begin{array}{lllllllll}0.205 & 0.741-0.100 & 0.873-0.1070 .819 & 0.036 & 0.939 & 0.228 & 0.273 & 0.424 & 0.035^{*}\end{array}$

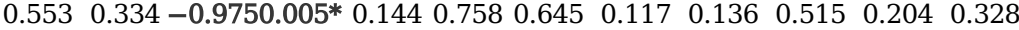

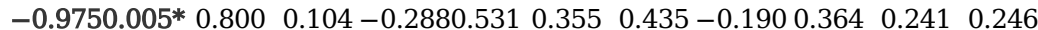

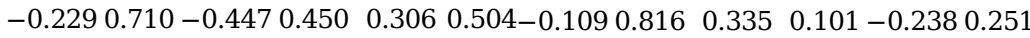

Lactobacillaceae/Lactobacillus

Firmicutes/Clostridia/Clostridiales

Lachnospiraceae

Peptostreptococcaceae

Clostridiaceae/Clostridium

Lachnospiraceae/Roseburia

Ruminococcaceae/Gemmiger

\begin{tabular}{lccc}
\hline Microbiota & Normal & Pre-obese & Obese \\
& $(n=5)$ & $(n=7)$ & $(n=25)$ \\
\cline { 2 - 4 }
\end{tabular}




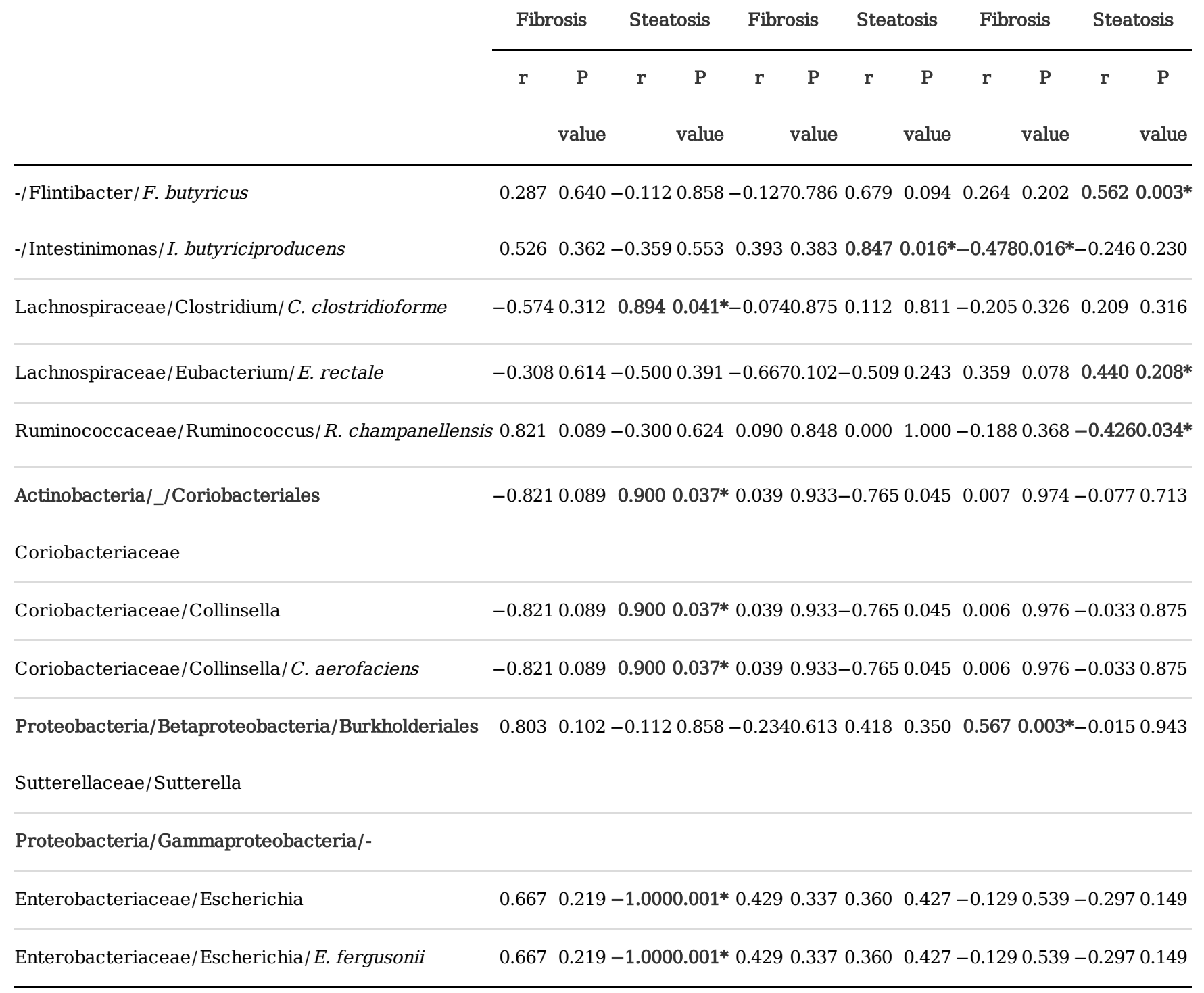

*Data are presented as the correlation coefficient (with an $\mathrm{r}$ value $0.4-0.59=$ medium, $0.6-0.79=$ strong, and $0.8-1=$ very strong correlation) and considered significant $(\mathrm{p}<0.05)$. The Spearman correlation test $(r)$ was used to evaluate the correlation between the Firmicutes/Bacteroidetes ratio and CAP and TE values.

\section{Figures}


NAFLD patients in Cipto Mangunkusumo Hospital (60 subjects)

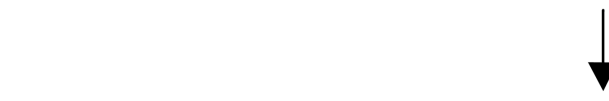

Food recall interview for the past month, inclusion and exclusion criteria (37 subjects)

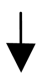

Data collection for other comorbidities

Physical examination: BMI

Additional examination: AST, ALT, HbA1c, lipid profile, fasting blood glucose, 2 hours postprandial blood glucose

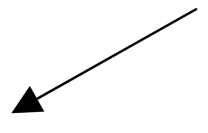

Fecal examination using $16 \mathrm{~S}$ rRNA metagenomic (NGS)
Controlled attenuation parameter (CAP) and Transient elastography (TE)

\section{Figure 1}

Subject recruitment and sample collection flow chart 
a
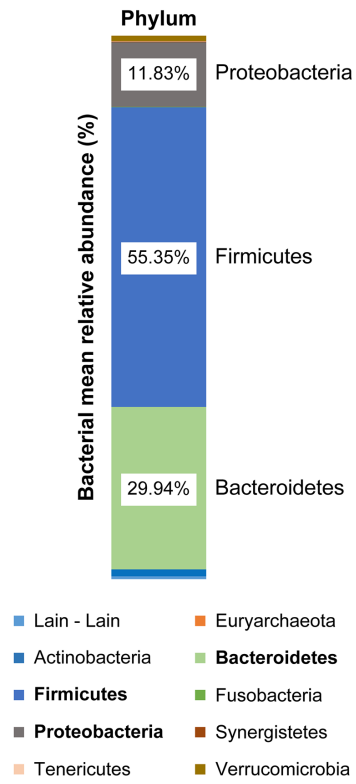

b

C
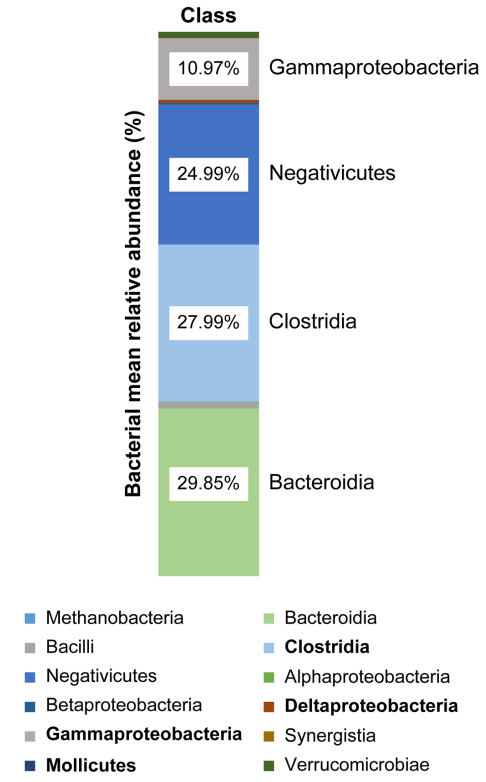

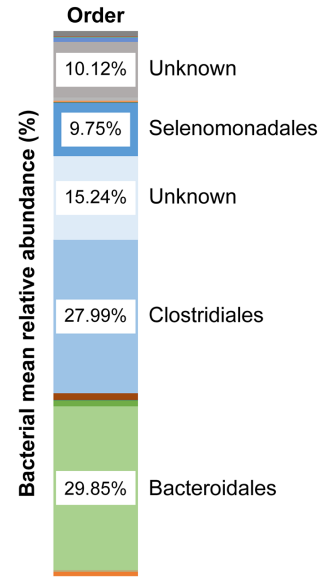

- Methanobacteriales - Bifidobacteriales - Coriobacteriales $\mid$ Sphingobacteriales = Bacteroidales Erysipelotrichales

- Bacillales Lactobacillales

- Clostridiales - -

- Selenomonadales Fusobacteriales

- Rhodospirillales Burkholderiales

- Desulfovibrionales =-

- Aeromonadales Pasteurellales d

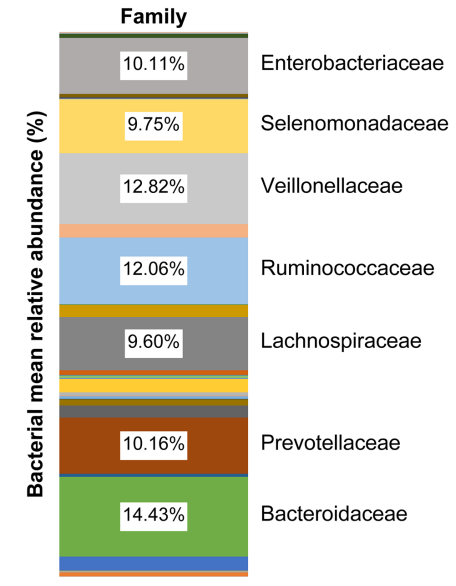

\begin{tabular}{|c|c|}
\hline Methanobacteriaceae & Bifidobacteriaceae \\
\hline Coriobacteriaceae & Sphingobacteriaceae \\
\hline- & Bacteroidaceae \\
\hline Porphyromonadaceae & - Prevotellaceae \\
\hline Rikenellaceae & Erysipelotrichaceae \\
\hline Bacillaceae & Enterococcaceae \\
\hline Lactobacillaceae & Leuconostocaceae \\
\hline Streptococcaceae & $=-$ \\
\hline Christensenellaceae & Clostridiaceae \\
\hline Clostridiales Family XIII. Incertae Sedis & Eubacteriaceae \\
\hline Lachnospiraceae & Oscillospiraceae \\
\hline - Peptococcaceae & - Peptostreptococcaceae \\
\hline Ruminococcaceae & Acidaminococcaceae \\
\hline Veillonellaceae & Selenomonadaceae \\
\hline Fusobacteriaceae & \#hodospirillaceae \\
\hline Comamonadaceae & - Oxalobacteraceae \\
\hline \# Sutterellaceae & Desulfovibrionaceae \\
\hline Enterobacteriaceae & - Succinivibrionaceae \\
\hline Pasteurellaceae & \#ynergistaceae \\
\hline
\end{tabular}

- Spiroplasmataceae

Figure 2

Characteristics of gut microbiota in bacterial mean relative abundance. a: Phylum; b: Class; c: Order; $d$ : Family 
a

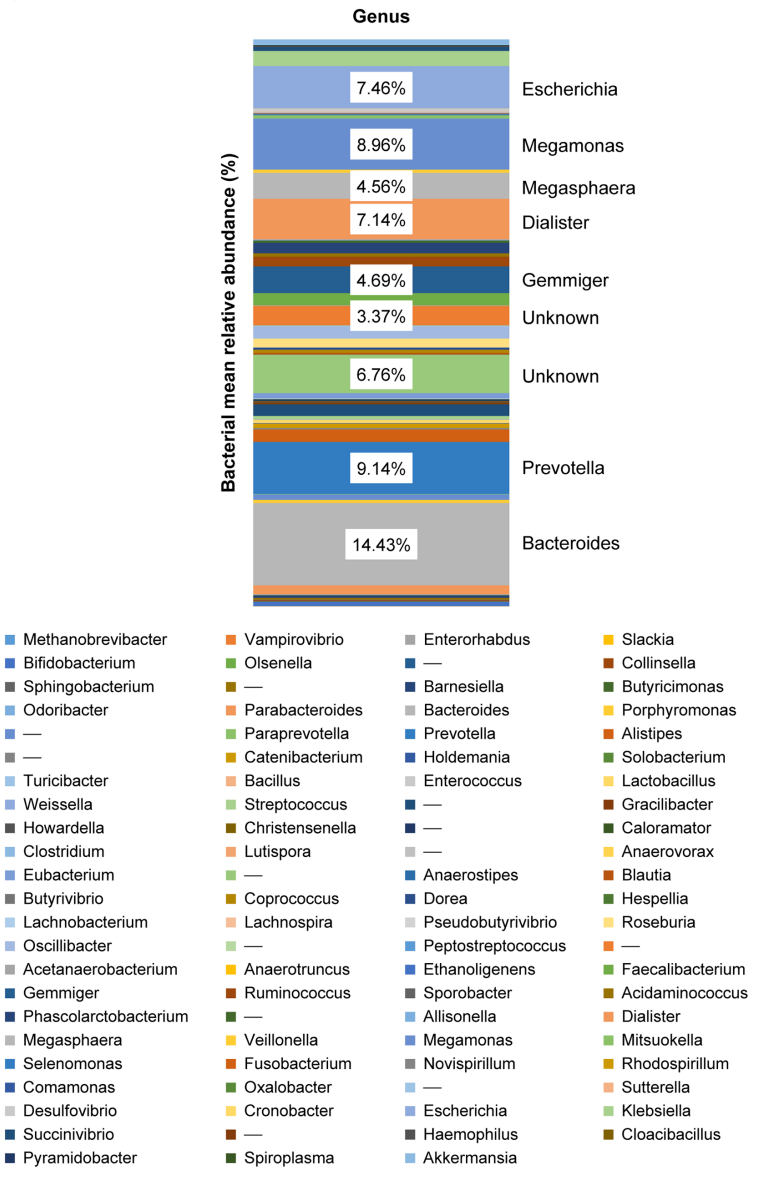

b

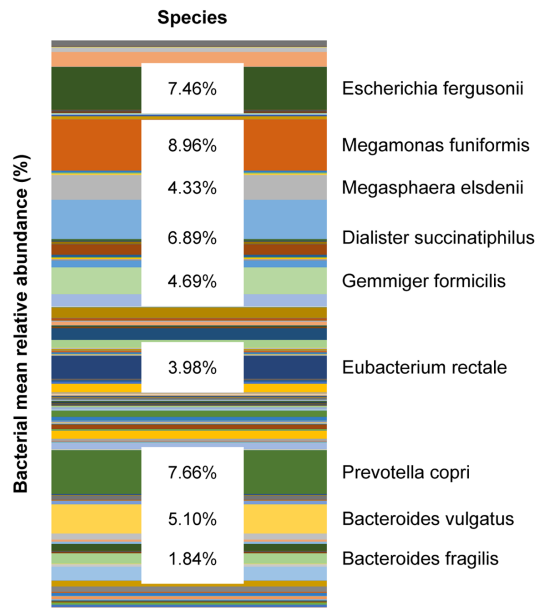

- Methanobrevibacter smithii - Bifidobacterium adolescentis - Senegalimassilia anaero - Odoribacter splanchnicus - Parabacteroides merda -Bacteroides coprocol

Bacteroides faecis

- Bacteroides massiliensis

Bacteroides stercoris

- Porphyromonas gulae

-Prevotellamassilia timonensis

-Prevotella denticola

- Prevotella stercorea

- Alistipes onderdonkii

- [Clostridium] spiroforme

- Turicibacter sanguinis

- Lactobacillus rogosae

- Streptococcus equinus

- Flavonifractor plautii

- Natranaerovirga pectinivora

-Closion celobioparum

-Christensenella massilie

Clostridium saudiense

- Eubacterium ruminantium

Abyssivirga alkaniphila

- Eisenbergiella tayi

- Eisenbergiella tayi

- Lachoclostridum pacaense

- [Clostridium] lactar

-Butyrivibrio crossotus
"Dorea formicigenerans

- Dorea formicigenerans
- Lachnobacterium bovis

Roseburia hominis

- Intestinibacter bartlettii

- Agathobaculum desmolans

- Negativibacillus massiliensis

- [Clostridium] leptum

- Acetanaerobacterium elongatum

- Faecalibacterium prausnitz

- Acidaminococcus intestini

- Allisonella histaminiformans

- Allisonella histaminifor

- Veillonella ratti

- Veillonella ratti

- Comamonas kerstersii

- Sutterella wadsworthensis

- Cronobacter dublinensis

- Succinivibrio dextrinosolven

- Pyramidobacter piscolens
- Vampirovibrio chlorellavorus Bifidobacterium longum - Collinsella aerofaciens - Barnesiella intestinihominis - Parabacteroides chinchillae - Bacteroides caccae -Bacteroides dorei

Bacteroides fragilis

- Bacteroides ovatus

- Bacteroides thetaiotaomicron

-Alloprevotella rava

- Paraprevotella clara

- Prevotella jejuni

Alistipes ihumii

Alistipes putredinis

- Catenibacterium mitsuoka

Bacillus zhangzhouensis

Lactobacillus ruminis

-Streptococcus parasanguinis

-Flintibacter butyricus

Pseudoflavonifractor phocaeensis

- [Clostridium] straminisolvens

- [Clostridium] hathewayi

Sarcina ventriculi

- Anaerovorax odorimutans

- Eubacterium ventriosum

Anaerocolumna cellulosilytica

- Frisingicoccus caecimuris

-[Clostridium] asparagiforme

[Clostridium] xylanolyticum

-Blautia luti

Dorea longicatena

Lachnospira pectinoschiza

Roseburia inulinivorans

Romboutsia timonensis

Anaerobacterium chartisolvens

Neglecta timonensis

[Clostridium] methylpentosum

Anaerotruncus colihominis

Gemmiger formicilis

Ruminococcus faecis

- Phascolarctobacter

Megasphaera massiliensis

Megamonas funiformis

Selenomonas ruminantium

Oxalobacter formigenes

Desulfovibrio desulfurican

Escherichia fergusonii

- Necropsobacter rosorum
Coprococcus catus
- Enterorhabdus muris

- Sphingobacterium mucilaginosum

- Butyricimonas faecihominis

- Parabacteroides distasoni

-Bacteroides caecimuris

Bacteroides eggerthii

- Bacteroides intestinalis

- Bacteroides paurosaccharolyticus

- Bacteroides uniformis

- Alloprevotella tannerae

- Prevotella colorans

Prevotella oralis

- Alistipes indistinctus

-Erysipelatoclostridium ramosum

Holdemania massiliensis

Enterococcus hirae

- Lactobacillus salivarius

- Streptococcus pasteurianus

-Intestinimonas butyriciproducens

Ruminiclostridium thermocellum

- Gracilibacter thermotolerans

w Caloramator australicus

- Lutispora thermophila

- Eubacterium coprostanoligenes

Anaerotaenia torlap

Fusicatenibacter saccharivorans

[Clostidium] clostridioform

- [Eubacterium] rect

Copro

- Coprococcus com

Pspellop poina

Oscubulyrivibrio ruminis

- Oscillibacter ruminantium

Antostreplococcus russellii

- Paludicola psychrotoleran

- [Clostridium] viride

Ancus rubiinfants

- Ruminococcus bromi

- Sporobacter termitidis

- Phascolarctobacterium

- Dialister succinatiphilus

-Veillonella atypica

Fusobacterium mortiferum

Fusobacterium mortiferum

- Parasutterella excre

- Desulfovibrio pige

Haemophilus parainfluenza
Hebsiella oxytoca

- Haemophilus parainfluenza
- Akkermansia muciniphila
-Eubacterium xylanophilum

Ana
= Slackia isoflavoniconverten - Olsenella umbonata - Coprobacter fastidiosus - Parabacteroides goldsteinii - Bacteroides chinchillae Bacteroides faecichinchilla - Bacteroides kribbi

- Bacteroides plebeius

Bacteroides vulgatus

- Massiliprevotella massiliensis

- Prevotella copri

Prevotella salivae

=Alistipes obesi

- Holdemanella biformis

- Solobacterium moorei

- Lactobacillus mucosae

-Weissella confusa

Colidextribacter massiliensis

- Monoglobus pectinilyticus

- Howardella ureilytica

Eubacterium ramulus

$=$ Anaerotignum aminivorans

= Kineothrix alysoides

- [Clostridium] colinum

- Anaerostipes hadrus

[Ruminococcus] gnavus

- Coprococcus eutactus

Roseburia faecis

- Oscillibacter valericigenes

- Agathobaculum butyriciproducen

-Fournierella massiliensis

- Ruthenibacterium lactatiformans

- [Eubacterium] siraeum

Ethanoligenens harbinense

Ruminococcus callidus

- Acidamnococcus fermentans

- Caecibacter massiliensis

" Megasphaera cerevisionella dispar
Veillone

Mitsuokella multacida

Mitsuokella multacida
Rhodospirillum rubrum

Rhodospirillum rubrum

Sutterella massiliensis

- Desulfovibrio simplex

Klebsiella pneumoniae
ECloacibacillus evryensis

\section{Figure 3}

Characteristics of gut microbiota in bacterial mean relative abundance. a: Genus; b: Species 